\title{
Motif-centric phosphoproteomics to target kinase-mediated signaling pathways
}

\author{
Chia-Feng Tsai ${ }^{1}$, Kosuke Ogata $^{1}$, Naoyuki Sugiyama ${ }^{1}$, Yasushi Ishihama ${ }^{1,2}$ \\ ${ }^{1}$ Graduate School of Pharmaceutical Sciences, Kyoto University, Kyoto 606-8501, Japan. \\ ${ }^{2}$ Laboratory of Clinical and Analytical Chemistry, National Institute of Biomedical Innovation, \\ Health and Nutrition, Ibaraki, Osaka, 567-0085, Japan.
}

*Correspondence: yishiham@pharm.kyoto-u.ac.jp

Tel: $+81-75-753-4555$

Fax: $+81-75-753-4601$

\section{Summary}

Identifying cellular phosphorylation pathways based on kinase-substrate relationships is a critical step to understanding the regulation of physiological functions in cells. Mass spectrometry-based phosphoproteomics workflows have made it possible to comprehensively collect information on individual phosphorylation sites in a variety of samples. However, there is still no generic approach to uncover phosphorylation networks based on kinase-substrate relationships in rare cell populations. Here, we describe a motif-centric phosphoproteomics approach combined with multiplexed isobaric labeling, in which in vitro kinase reaction is used to generate the targeted phosphopeptides, which are spiked into one of the isobaric channels to increase detectability. Proof-of-concept experiments demonstrate selective and comprehensive quantification of targeted phosphopeptides by using multiple kinases for motif-centric channels. Over 7,000 tyrosine phosphorylation sites were quantified from several tens of $\mu \mathrm{g}$ of starting materials. This approach enables the quantification of multiple phosphorylation pathways under physiological or pathological regulation in a motif-centric manner.

Keywords: Motif-centric, Phosphoproteome, Kinase, IMAC

Motivation: Sensitivity for detecting phosphopeptides with a particular phosphorylation motif is limited, especially for tyrosine phosphopeptides. 


\section{Introduction}

Protein kinase-mediated phosphorylation on serine, threonine and tyrosine residues is one of the most ubiquitous post-translational modifications (PTMs). Signaling cascades via protein phosphorylation play key roles in multiple cellular processes in mammals, including intra- and intercellular signaling, protein synthesis, gene expression, cell survival and apoptosis (Cohen, 2002; Hunter, 2000). The relative abundances of phosphoserine (pS), phosphothreonine (pT), and phosphotyrosine ( $\mathrm{pY}$ ) sites in the human proteome have been estimated to be 90:10:0.05 based on the traditional method of ${ }^{32} \mathrm{P}$ labeling (Hunter and Sefton, 1980). There are many possible reasons for the extreme paucity of $\mathrm{pY}$ sites compared to $\mathrm{pS}$ and $\mathrm{pT}$ sites in mammals, including the fact that tyrosine kinases are activated only under certain conditions, and that the high activity of protein tyrosine phosphatase (PTP) leads to a short half-life of pY sites (Hunter, 2009).

Great advances in the analytical workflows of shotgun phosphoproteomics, in which metal affinity chromatography is integrated with LC/MS/MS, have made it possible to identify more than 30,000 phosphorylation sites (Bekker-Jensen et al., 2017; Hogrebe et al., 2018; Mertins et al., 2018). In general, LC/MS/MS has an identification bias toward the more abundant phosphopeptides in a sample, whereas in kinase substrates, sequence features such as Prodirected, basophilic, acidophilic and tyrosine-containing motifs have an important influence (Villen et al., 2007). Also, since biological importance does not necessarily correlate with protein expression levels, it is possible that important signals are transduced via specific kinases that are expressed at extremely low levels. Therefore, advanced pre-fractionation or enrichment methods prior to LC/MS/MS are needed to identify a wide range of kinase substrates (Tsai et al., 2014). Especially for low-abundance pY peptides, MS detectability is affected by the ionization suppression caused by the presence of more abundant $\mathrm{pS}$ and $\mathrm{pT}$ peptides in the complex phosphoproteomes. However, the combination of metal-affinity chromatography with immunoaffinity purification using pY antibody (Abe et al., 2017) or a recently developed SH2 domain-derived pY-superbinder (Bian et al., 2016; Dong et al., 2017) has been reported to increase the identification number of $\mathrm{pY}$ peptides. In addition, immunoaffinity-based methods using multiple antibodies have been developed for identification and quantitation of phosphopeptides derived from proteins in various pathways or pY peptides derived from tyrosine kinases (Stokes et al., 2012). Nevertheless, a large amount of starting material ( $\mathrm{mg}$ to ten $\mathrm{mg}$ level) is generally necessary for deep tyrosine phosphoproteome analysis.

One of the major advantages of multiplexed isobaric tandem mass tag (TMT)-based methods for relative quantitation is that the differentially labeled peptides appear as a single peak at the MS1 level (Thompson et al., 2003), enhancing the detectability of low-abundance peptides. TMT strategies using a large amount of relevant "boosting" (or "carrier") peptides labeled with one or several TMT channels have been successfully used for single-cell proteomics analysis (Budnik et al., 2018; Dou et al., 2019; Tsai et al., 2020). For instance, Lian et al. developed a BASIL (Boosting to Amplify Signal with Isobaric Labeling) strategy to quantify $>20,000$ phosphorylation sites in human pancreatic islets. However, the 
identification number of $\mathrm{pY}$ peptides was less than $1 \%$. Recently, Chua et al. developed a BOOST (Broad-spectrum Optimization Of Selective Triggering) method in which pervanadate (tyrosine phosphatase inhibitor)-treated cells were used as a boosting channel to increase the detectability of pY peptides (Chua et al., 2020). The BOOST method coupled with antibodybased pY enrichment could quantify over 2300 unique pY peptides. However, the required amount of starting material was in the mg range, making it difficult to apply to small samples such as clinical specimens, which often contain less than $100 \mu \mathrm{g}$ of extractable material.

We previously developed an LC/MS/MS-based in vitro kinase assay using dephosphorylated lysate proteins as the substrate source for in vitro kinase reaction to profile human protein kinomes (Imamura et al., 2014). A total of 175,574 direct kinase substrates were identified from 354 wild-type protein kinases, 21 mutant protein kinases, and 10 lipid kinases (Sugiyama et al., 2019). In addition, we utilized the in vitro kinase reactions with CK2, MAPK and EGFR to generate phosphopeptides with targeted motifs to measure the phosphorylation stoichiometry of $>1,000$ phosphorylation sites, including 366 low-abundance tyrosine phosphorylation sites (Tsai et al., 2015).

In the present study, we aimed to develop a motif-centric TMT approach in which phosphopeptides having targeted sequence motifs are generated by in vitro kinase reaction for the boosting TMT channel in order to increase the detectability of kinase substrates, including tyrosine kinase substrates, without immunoaffinity enrichment. To demonstrate the feasibility of this strategy, phosphopeptides with targeted motifs of CK2, PKA, CDK1, ERK2, JNK1, p38a, SRC and EGFR were used for the boosting TMT channel to monitor the perturbation of kinases-mediated signaling pathways by tyrosine kinase inhibitor treatment.

\section{STAR $\star$ Methods}

\begin{tabular}{|l|l|l|}
\hline REAGENT or RESOURCE & SOURCE & IDENTIFIER \\
\hline $\begin{array}{l}\text { Biological Samples } \\
\text { HeLa S3 }\end{array}$ & ATCC & Catalog: CCL-2.2 \\
\hline $\begin{array}{l}\text { Critical Commercial Reagents } \\
\text { and Assays }\end{array}$ & & \\
\hline Triethylammonium bicarbonate & Sigma & Catalog: T7408 \\
\hline Phosphatase Inhibitor Cocktail 2 & Sigma & Catalog: P5726 \\
\hline Phosphatase Inhibitor Cocktail 3 & Sigma & Catalog: P0044 \\
\hline BCA Protein Assay Kit & Thermo Scientific & \\
\hline Sodium deoxycholate & Pierce & Catalog: 23225 \\
\hline Sodium lauroyl sarcosinate & FUJIFILM Wako & Catalog: 190-08313 \\
\hline Iron-(III) chloride & FUJIFILM Wako & Catalog: 198-14745 \\
\hline Dithiothreitol & FUJIFILM Wako & Catalog: 091-00872 \\
\hline lodoacetamide & Thermo Scientific & Catalog: 20291 \\
\hline Lysyl endopeptidase & Thermo Scientific & Catalog: A3221 \\
\hline & FUJIFILM Wako & Catalog: 129-02541 \\
\hline
\end{tabular}




\begin{tabular}{|l|l|l|}
\hline $\begin{array}{l}\text { Sequencing-grade modified } \\
\text { trypsin }\end{array}$ & Promega & Catalog: V517 \\
\hline Ni-NTA silica resins & QIAGEN & Catalog: 31314 \\
\hline Empore SDB-XC membrane disks & CDS & Catalog: 13-110-020 \\
\hline CK2 $\alpha 1 / \beta(C S N K 2 A 1 / B)$ & Carna Biosciences & Catalog:05-184 \\
\hline CK2 $\alpha / \beta(C S N K 2 A 2 / B)$ & Carna Biosciences & Catalog:05-185 \\
\hline PKAC $\alpha($ PRKACA) & Carna Biosciences & Catalog:01-127 \\
\hline ERK2 (MAPK1) & Carna Biosciences & Catalog:04-143 \\
\hline EGFR (ERBB1) & Carna Biosciences & Catalog:08-115 \\
\hline SRC & Carna Biosciences & Catalog:08-173 \\
\hline JNK1(MAPK8) & Carna Biosciences & Catalog:04-163 \\
\hline CDK1 (CDC2/CycB1) & Carna Biosciences & Catalog:04-102 \\
\hline p38 $\alpha(M A P K 14)$ & Carna Biosciences & Catalog:04-152 \\
\hline TMTsixplex & Thermo Scientific & Catalog:90061 \\
\hline & & \\
\hline Software and Algorithms & & \\
\hline Maxquant & PMID: 27809316 & https://www.maxquant.org/ \\
\hline Perseus & PMID: 27348712 & https://maxquant.net/perseus/ \\
\hline & & \\
\hline Deposited Data & & \\
\hline jPOST Repository & PMID: 30295851 & https://repository.jpostdb.org \\
\hline
\end{tabular}

\section{Tryptic peptides from HeLa cell lysate}

HeLa S3 cells were cultured in DMEM containing $10 \% \mathrm{FBS}$ and $100 \mu \mathrm{g} / \mathrm{mL}$ kanamycin. For isobaric acidophilic motif-centric phosphoproteomes, cells were not stimulated (mock) or were stimulated with $10 \mu \mathrm{M}$ CK2 inhibitor (CX-4945) for 30 mins. For isobaric basophilic motifcentric phosphoproteomes, cells were not stimulated (mock) or were stimulated with $10 \mu \mathrm{M}$ PKA activator (forskolin) for 30 mins. For isobaric tyrosine and multiple motif-centric phosphoproteome, cells were treated with $10 \mu \mathrm{M}$ EGF, $10 \mu \mathrm{M}$ EGF/10 $\mu \mathrm{M}$ afatinib and 500 $\mu \mathrm{M}$ pervanadate $\left(\mathrm{pH} 10\right.$ with $\left.0.14 \% \mathrm{H}_{2} \mathrm{O}_{2}\right)$, respectively, for 30 mins before harvesting. Two biological replicates were performed. Cells were washed three times with ice-cold phosphatebuffered saline (PBS, $0.01 \mathrm{M}$ sodium phosphate, $0.14 \mathrm{M} \mathrm{NaCl}, \mathrm{pH} 7.4$ ) and then lysed in lysis buffer containing $12 \mathrm{mM}$ sodium deoxycholate (SDC), $12 \mathrm{mM}$ sodium lauroyl sarcosinate (SLS) in $100 \mathrm{mM}$ triethylammonium bicarbonate (TEABC). Protein concentration was determined by means of BCA protein assay. The lysates were digested based on the reported phasetransfer surfactants (PTS) protocol (Masuda et al., 2008). The digested peptides were desalted on SDB-XC StageTips (Rappsilber et al., 2007).

\section{In vitro kinase reaction}

For acidophilic, Pro-directed and tyrosine kinase reactions, the tryptic peptides were dissolved in $40 \mathrm{mM}$ Tris- $\mathrm{HCl}(\mathrm{pH} \mathrm{7.5)}$ and incubated with each kinase $(0.2 \mu \mathrm{g}$ CK2, ERK2, JNK1, p38 $\alpha$, CDK1 or SRC) at $37{ }^{\circ} \mathrm{C}$ for in vitro kinase reaction in the presence of $1 \mathrm{mM}$ ATP and 20 $\mathrm{mM} \mathrm{MgCl}$. For EGFR kinase reaction, tryptic peptides were firstly passed through $\mathrm{SCX}$ 
StageTips (Rappsilber et al., 2007) to remove afatinib. Eluted peptides were further desalted on SDB-XC StageTips. Then, the desalted peptides were dissolved in $40 \mathrm{mM}$ Tris- $\mathrm{HCl}(\mathrm{pH} 7.5)$ and incubated with EGFR $(0.2 \mu \mathrm{g})$ for in vitro kinase reaction in the presence of $1 \mathrm{mM}$ ATP and $4 \mathrm{mM} \mathrm{MnCl} 2$ at $37^{\circ} \mathrm{C}$. For basophilic kinases such as PKA, the lysates were loaded onto a 10 kDa ultrafiltration device (Amicon Ultra, Millipore). The device was centrifuged at $14,000 \mathrm{~g}$ to remove the detergents. Subsequently, the original lysis buffer was replaced with $40 \mathrm{mM}$ Tris$\mathrm{HCl}(\mathrm{pH} \mathrm{7.5)}$ followed by centrifugation. Then, the proteins were incubated with $0.2 \mu \mathrm{g}$ PKA for in vitro kinase reaction in the presence of $1 \mathrm{mM}$ ATP and $20 \mathrm{mM} \mathrm{MgCl}_{2}$. After the kinase reaction, the proteins were reduced with $10 \mathrm{mM}$ DTT for 30 mins at $37^{\circ} \mathrm{C}$ and alkylated with $50 \mathrm{mM}$ iodoacetamide in the dark for $30 \mathrm{mins}$ at $37^{\circ} \mathrm{C}$. The resulting samples were digested by Lys-C $(1: 100, \mathrm{w} / \mathrm{w})$ at $37^{\circ} \mathrm{C}$ for $3 \mathrm{hr}$ followed by trypsin $(1: 50, \mathrm{w} / \mathrm{w})$ overnight at $37^{\circ} \mathrm{C}$. All the peptides were desalted on SDB-XC StageTips.

\section{TMT labeling for purified phosphopeptides}

The desalted peptides were dissolved in $200 \mathrm{mM}$ HEPES ( $\mathrm{pH}$ 8.5). Then, the resuspended phosphopeptides were mixed with TMT reagent dissolved in 100\% ACN for $1 \mathrm{hr}$. The labeling reaction was stopped by adding $5 \%$ hydroxylamine for 15 minutes, followed by acidification with TFA. All the phosphopeptides labeled with each multiplexed TMT reagent were mixed into the same tube and the mixture was diluted to reduce the concentration of ACN to below $5 \%$. The TMT-labeled peptides were desalted on SDB-XC StageTips.

\section{Immobilized metal ion affinity chromatography (IMAC)}

The procedure for phosphopeptides purification with an $\mathrm{Fe}^{3+}-\mathrm{IMAC}$ tip was as described previously (Tsai et al., 2014; Tsai et al., 2015) with minor modifications. In brief, buffer consisting of $50 \mathrm{mM}$ EDTA in $1 \mathrm{M} \mathrm{NaCl}$ was used for removing $\mathrm{Ni}^{2+}$ ions. Then, the metal-free NTA was activated by loading $100 \mathrm{mM} \mathrm{FeCl}_{3}$ into the IMAC tip. The $\mathrm{Fe}^{3+}$-IMAC tip was equilibrated with $1 \%(\mathrm{v} / \mathrm{v})$ acetic acid at $\mathrm{pH} 3.0$ prior to sample loading. Tryptic peptides from HeLa lysates were reconstituted in $1 \%(\mathrm{v} / \mathrm{v})$ acetic acid and loaded onto the IMAC tip. After successive washing steps with $1 \%(\mathrm{v} / \mathrm{v})$ TFA in $80 \% \mathrm{ACN}$ and $1 \%(\mathrm{v} / \mathrm{v})$ acetic acid, the IMAC tip was inserted into an activated SDB-XC StageTip. Then, the bound phosphopeptides were eluted into the SDB-XC StageTip by adding $200 \mathrm{mM} \mathrm{NH}_{4} \mathrm{H}_{2} \mathrm{PO}_{4}$. The eluted phosphopeptides were desalted on the SDB-XC StageTip.

\section{$\underline{L C / M S / M S \text { analysis }}$}

NanoLC/MS/MS analyses were performed on an Orbitrap Fusion Lumos Tribrid mass spectrometer (Thermo Scientific, San Jose, CA), which was connected to a Thermo Ultimate 3000 RSLCnano system (Germering, Germany) and an HTC-PAL autosampler (CTC Analytics, Zwingen, Switzerland). Peptide mixtures were loaded onto and separated on self-pulled needle columns (150 mm length $\times 100 \mu \mathrm{m}$ ID) packed with Reprosil-Pur 120 C18-AQ material (3 $\mu \mathrm{m}$, Dr. Maisch GmbH, Amerbuch, Germany) or a 2-m long C18 monolithic silica capillary column (Iwasaki et al., 2010). The mobile phases consisted of (A) $0.5 \%$ acetic acid and (B) $0.5 \%$ 
acetic acid and $80 \%$ acetonitrile. Peptides were separated through a gradient from $17.5 \%$ to $45 \%$ buffer $B$ at a flow rate of $500 \mathrm{~nL} / \mathrm{min}$. Full-scan spectra were acquired at a target value of $4 \times 10^{5}$ with a resolution of 60,000 . Data were acquired in a data-dependent acquisition mode using the top-speed method $(3 \mathrm{sec})$. The peptides were isolated using a quadrupole system (the isolation window was 0.7 ). The $\mathrm{MS}^{2}$ analysis was performed in the ion trap using CID fragmentation with a collision energy of 35 at a target value of $1 \times 10^{4}$ with $100 \mathrm{mS}$ maximum injection time. The $\mathrm{MS}^{3}$ analysis was performed for each $\mathrm{MS}^{2}$ scan acquired by using multiple $\mathrm{MS}^{2}$ fragment ions isolated by an ion trap as precursors for the $\mathrm{MS}^{3}$ analysis with a multinotch isolation waveform(McAlister et al., 2014). HCD fragmentation was used for $\mathrm{MS}^{3}$ scan with an NCE of $65 \%$, and the fragment ions were detected by the Orbitrap (resolution 15000). The AGC target was $5 \times 10^{4}$ with a maximum ion injection time of $22 \mathrm{~ms}$. The raw data sets have been deposited at the ProteomeXchange Consortium (http://proteomecentral.proteomexchange.org) via the jPOST partner repository (http://ipostdb.org) (Moriya et al., 2019) with the dataset identifier JPST001027 (PXD026996).

\section{Database search}

The raw MS/MS data were processed with MaxQuant (Cox and Mann, 2008; Tyanova et al., 2016a). Peptide search with full tryptic digestion and a maximum of two missed cleavages was performed against the SwissProt human database (20,102 entries). The mass tolerance for precursor and $\mathrm{MS}^{3}$ ions was $4.5 \mathrm{ppm}$, whereas the tolerance for $\mathrm{MS}^{2}$ ions was $0.5 \mathrm{Th}$. Acetylation (protein $\mathrm{N}$-terminal), oxidation (M) and phospho (STY) were set as variable modifications and carbamidomethyl (C) was set as a fixed modification. The quantitation function of reporter ion $\mathrm{MS}^{3}$ (6-plexed TMT) was turned on. The false-discovery rate (FDR) was set to $1 \%$ at the level of PSMs and proteins. A score cut-off of 40 was used for identified modified peptides. The abundances of TMT were $\log _{2}$-transformed and further analyzed by Perseus (Tyanova et al., 2016b) for statistical evaluation. The PSP logo generator (Hornbeck et al., 2015) was used for sequence motif analysis.

\section{Results}

\section{Workflow for isobaric motif-centric phosphoproteome analysis}

We previously developed a motif-centric approach (Tsai et al., 2015) in which dephosphorylation and isotope tagging are integrated with in vitro kinase reaction to improve the sensitivity and reproducibility for determining the absolute phosphorylation stoichiometry of targeted kinase substrates. However, the number of phosphosites commonly identified in endogenous and motif-centric phosphopeptides was not as large as expected, because some endogenous signals with specific kinase motifs are below the detection limit. In this study, we employed not isotopic but isobaric tagging to develop a motif-centric approach in which the same peptides from different samples were labeled with multiplexed TMT reagents and assembled as a single peak at the $\mathrm{MS}^{1}$ level to increase the sensitivity. In addition, we set one of the TMT channels for signal boosting, utilizing phosphopeptides having targeted sequence motifs generated by in vitro kinase reaction to 
increase the detectability of targeted kinase substrates. The entire workflow is shown in Figure 1 . The motif-centric peptides are generated by in vitro kinase reactions using Prodirected, acidophilic, basophilic or tyrosine kinase (Figure 1a). The same biological resource (same cell type or tissue) can be used to accomplish the back-phosphorylation (Li et al., 2016; Mundina-Weilenmann et al., 1991) without any pre-dephosphorylation process(Tsai et al., 2015). In vitro kinase reactions both at the protein and tryptic peptide levels can be used in most cases, except for basophilic kinase reactions, where tryptic peptides cannot be used as substrates due to the lack of $\mathrm{K}$ or $\mathrm{R}$ at the $\mathrm{N}$-terminal side of the phospho accepting sites. In such a case, the kinase reaction at the protein level, followed by tryptic digestion, is used to generate the basophilic motif-centric phosphopeptides. After TMT labeling, phosphopeptides are enriched by metal affinity chromatography and analyzed by nanoLC/MS/MS (Figure 1b). The TMT-labeled precursor ions from the endogenous and back-phosphorylated peptides are fragmented and the assembled signals in b or $y$ ions are helpful for peptide identification, especially for endogenous phosphopeptides with low abundance. The relative quantitation of endogenous phosphopeptides across different samples is done by the use of reporter ions at the $\mathrm{MS}^{3}$ level, by the implementation of synchronous precursor selection (SPS)-based $\mathrm{MS}^{3}$ technology (McAlister et al., 2014), which can reduce the interference signals due to coisolation of precursor ions.

\section{Acidophilic motif-centric phosphoproteome analysis}

We firstly employed the motif-centric TMT approach to quantify the kinase-perturbed phosphorylation changes in HeLa cells using CK2 kinase inhibitor (CKi, Silmitasertib, CX-4945). The tryptic peptides from CKi-treated HeLa cells were phosphorylated by CK2 in vitro and labeled with both $\mathrm{TMT}^{128}$ and $\mathrm{TMT}^{131}$ for $\mathrm{CK} 2$ motif-centric boosting channels. The phosphopeptides from DMSO-treated cells were labeled with both $\mathrm{TMT}^{126}$ and $\mathrm{TMT}^{129}$, and phosphopeptides from CKi-treated cells were labeled with both $\mathrm{TMT}^{127}$ and $\mathrm{TMT}^{130}$. An MS spectrum and an MS ${ }^{3}$ spectrum of a known CK2 substrate, the pS66 site in LIG1, are shown in Figure 2. After fragmentation by $\mathrm{CID}$, the peptide sequence and phosphosite localization information can be annotated at the $\mathrm{MS}^{2}$ level (Figure 2a). Then, the $\mathrm{MS}^{3}$ spectrum was obtained, demonstrating that the TMT signals of endogenous phosphopeptides were decreased after the CKi treatment (TMT ${ }^{127}$ and $\mathrm{TMT}^{130}$ in Figure $2 \mathrm{~b}$ ) and that the TMT signals of CK2-motif targeting phosphopeptides were increased after in vitro kinase reaction (TMT ${ }^{128}$ and $\mathrm{TMT}^{131}$ in Figure $2 \mathrm{~b}$ ). These results indicate that this isobaric motif-centric approach can be used to monitor CK2 phosphorylation signaling in terms of the TMT ratios.

In total, this isobaric motif-centric approach quantified 4559 unique phosphopeptides (91\% specificity in phosphopeptide enrichment) from $25 \mu \mathrm{g}$ of starting material. The logarithm of the median ratio of CKi-treated to untreated peptides was negative, while the logarithm of the median ratio of motif-centric (CK2-motif) to CKi-treated peptides was positive and this increase was larger than the decrease caused by CKi treatment (Figure 2c), indicating that the in vitro kinase reaction effectively increased the TMT signals of peptides directly phosphorylated by CK2. Silmitasertib has been used to exclusively inhibit CK2 activity in 
previous studies(Chon et al., 2015; Wang et al., 2017). Therefore, we can discriminate direct CK2 substrates from others by comparison of TMT ratios such as CKi/DMSO and CK2-motif/CKi using Student's t-test. Based on the CKi/DMSO ratios, 66 (1.6\%) up-regulated and 750 (18\%) down-regulated phosphorylation sites were identified (Figure 2d). Among the downregulated phosphorylation sites, up to $35 \%(n=266)$ were significantly increased after in vitro kinase reaction (group B in Figure 2d). After sequence motif analysis (O'Shea et al., 2013), we found that the sequence motif of the phosphopeptides in group $B$ agreed with the known CK2 substrate motif (acidic motif), whereas the motif logo from group A contained both Pro and Asp at +1 position (Figure $2 d$ ). Among the phosphorylation sites $(n=266)$ in group $B, 222$ sites were registered in a public phosphorylation sites database (Hornbeck et al., 2015), including 6 known CK2 substrates: PTGES3 (S113), LIG1 (S66), SLC3A2 (S375), CDK1 (S39), TOP2A (S1377) and ABCF1(S110). For group B proteins, we also examined the overlap with CK2 interactors in STRING and found 20 known CK2 interactors (Figure S1a). We further performed Gene Ontology and Reactome Pathway enrichment analysis for group B proteins(Szklarczyk et al., 2019). As a result, 137 proteins in group B were annotated as nuclear proteins, and a majority of them possessed functions related to ATP binding, DNA binding, nucleotide binding and so on (Table S1). Among the annotated pathways, the top hit was the cell cycle pathway, in which 21 group $B$ proteins were down-regulated, including CDK1, a known CK2 substrate that affects cell cycle regulation upon S39 phosphorylation(Bloom and Cross, 2007; Russo et al., 1992) (Figure S1b). All these results indicate that this motif-centric approach is an effective tool for monitoring specific kinase-mediated signaling pathways.

\section{Basophilic motif-centric phosphoproteome analysis}

The isobaric motif-centric approach was further examined with PKA as a basophilic kinase, using forskolin as an activator. Unlike acidophilic CK2, PKA cannot be used to generate motifcentric peptides from tryptic peptides because it requires basic amino acid residues at the $\mathrm{N}$ terminal side of the phospho-acceptor. Therefore, PKA motif-centric peptides were generated by in vitro kinase reaction at the protein level, followed by tryptic digestion (Fig. 1a). After the isobaric labeling, the TMT ratios of three channels, forskolin-treated, DMSO-treated and motif-centric samples, were used to discriminate the peptides phosphorylated directly by PKA from others, as in the case of CK2. The results are illustrated in Figure S2. In total, we quantified 7855 phosphorylation sites from biological replicate experiments. Among them, 620 phosphorylation sites were upregulated (group 1) and 322 phosphorylation sites were inhibited by forskolin (group 2). We previously established a computational model (primary sequence preference, PSP score) to characterize the kinase sequence specificity toward the substrate target site based on known kinase-substrate relationships, in order to exclude potential indirect targets of PKA (Imamura et al., 2017). We applied PSP scoring to the phosphorylation sites in groups 1 and 2, and found that the PSP scores of group 1 were significantly higher than those of group 2, although the sequence motifs of phosphorylation sites in groups 1 and 2 both belonged to the basophilic category (Figure S2b). Based on the 
above results, the motif-centric approach was able to discriminate peptides phosphorylated directly by PKA from indirectly phosphorylated ones.

\section{Tyrosine motif-centric phosphoproteome analysis}

Although the detectability of $\mathrm{pY}$ sites has been restricted by their extremely low phosphorylation stoichiometry compared to pS and pT sites(Sharma et al., 2014; Tsai et al., 2015), this lower stoichiometry results in a larger amount of unphosphorylated counterparts, which can be used for back-phosphorylation. In order to increase the identification number of pY sites, we firstly tried to use pervanadate (PV)-treated Hela cells as a pY-centric sample (Figure S3). PV is well-known as a tyrosine phosphatase inhibitor causing an increase in the stoichiometry of endogenous pY sites(Sharma et al., 2014). As expected, the pY content was increased from $0.8 \%$ to $11.7 \%$ (Figure S4a) by PV treatment. Then we employed the PVtreated peptides as pY-centric peptides with $25 \mu \mathrm{g}$ of untreated HeLa peptides. Indeed, the number of quantifiable $\mathrm{pY}$ sites increased from 74 to 595 as the spiking amount of the $\mathrm{pY}$ centric peptides was increased from 0 to $75 \mu \mathrm{g}$, without any antibody-based enrichment (Figure S4b). The amplified signals caused by the spiked $\mathrm{pY}$ peptides resulted in a much larger number of identified $\mathrm{pY}$ sites than in usual phosphoproteome analysis. However, the number of identified pY sites was still lower than that obtained using the recently published antibodybased boosting strategy (Chua et al., 2020), which also used PV-treated cells as boosting samples to quantify over 2300 unique pY peptides from $1 \mathrm{mg}$ of starting materials.

In order to extend the tyrosine phosphoproteome coverage, the back-phosphorylation sites of the untreated HeLa peptides were phosphorylated via in vitro kinase reaction by using tyrosine kinases such as EGFR and SRC. Note that EGFR and SRC have different phosphorylation motifs (Sugiyama et al., 2019). Then, the kinase-treated peptides were labeled with one of the 6-plexed TMT reagents to detect the endogenous pY sites in EGF or EGF/afatinib (EGFR inhibitor)-treated Hela cells (Figure S3). After IMAC enrichment, the TMTlabeled phosphopeptides were analyzed on a 2-m long monolithic silica column system with the SPS-MS ${ }^{3}$ technique (McAlister et al., 2014). Compared with the result from PV-treated HeLa as pY motif-centric peptides, the content of pY increased from $15 \%$ to $87 \%$ (Figure 3a) which resulted in an approximately 10 -fold increase in the numbers of identified and quantified Class 1 pY sites (localization probability $>0.75$ ), as shown in Figure $3 b$. However, Cheung et al. (Cheung et al., 2021) reported that the isobaric labeling-based quantitative approaches have technical limitations that potentially affect data quality and biological interpretation, due to the large amounts of spiked carrier samples. In addition, it is difficult to control the TMT ratio within the quantifiable range because the phosphorylation stoichiometry in cells depends on each pY site. Furthermore, we should reject TMT-peptides without reporter ion signals in the sample channels. Therefore, we examined the distribution of the TMT reporter ion intensity of each sample channel and found a notch to discriminate the signal from the noise (Figure S5); this was also mentioned in the previous study (Hughes et al., 2017). Based on this observation, we set the acceptance criterion for the minimum TMT intensity in the sample channels to be greater than 40 on a $\log _{2}$ scale for the total TMT 
intensity of the sample channels. We also set another criterion, that the maximum TMT ratio of the motif-centric channel to the sample channel should be less than 100 (Figure 3c). By applying these two criteria, we found that 668,5425 and $3688 \mathrm{pY}$ sites could be quantified in the PV-treated, EGFR-centric and SRC-centric samples, respectively. Among these $\mathrm{pY}$ sites, the sequence motifs of unique pY sites in each dataset were different (Figure 3d), which indicated that complementary $\mathrm{pY}$ sites can be identified by using different motif-centric peptides. For quantitation based on the TMT reporter ion intensities, the EGF and EGF-TKI treated cells were separated in the PCA analysis (Figure 3e). The above results demonstrate that this motifcentric approach through spiking isobaric tyrosine phosphopeptides with high purity was able to increase the detectability of $\mathrm{pY}$ sites without the need for any affinity purification procedure, such as application of $\mathrm{pY}$ antibody.

\section{Multiple motifs-centric approach to depict perturbed phosphoproteome}

We further used multiple kinases to generate a wide variety of motif-centric peptides to monitor the phosphorylation signals generated by different kinases. For proof-of-concept, we analyzed the phosphoproteome of EGF-treated and EGF/afatinib co-treated HeLa cells. We selected four Pro-directed kinases ((ERK1 (MAPK3), JNK1(MAPK8), p38 $\alpha$ (MAPK14) and CDK1)), two tyrosine kinases (SRC and EGFR) and one acidophilic kinase (CK2) to detect the different motif-centric phosphorylation sites (Figure S6). After filtering based on the boosting ratio (<100x) and TMT intensity cut-off (sum of TMT intensity from sample channels $>40$ on $\log 2$ scale), up to 11,895 class 1 phosphorylation sites were quantified (at least two valid TMT values in one cell type) including 5,045 pS, 1,756 pT and 5,094 pY sites (Figure 4a). According to Student's t-test (EGF vs. EGF/afatinib), the ratio of commonly regulated phosphorylation sites was consistent between experiments using different kinases, regardless of the kinase used (Figure 4b), indicating that the boosting channel did not significantly affect the quantitative results.

In addition to identifying which phosphorylation sites were regulated after afatinib treatment, we were also able to estimate which kinases phosphorylated which sites by using this motif-centric approach. For example, up to 18 class 1 phosphorylation sites, including 7 pY sites, on EGFR kinase were quantified without any immunoprecipitation step from $25 \mu \mathrm{g}$ of starting materials per TMT channel (Figure 4c). Among these sites, pY1144 is a known autophosphorylation site which was downregulated by afatinib and detected in the EGFR motif-centric experiment. This result indicates that the activity of this phosphorylation site is controlled by EGFR and also inhibited by afatinib. On the other hand, pS1051 and pS992 were downregulated by afatinib, but there is no information about the corresponding kinase in the public database. Through this motif-centric approach, we could determine that pS1051 and pS992 are likely phosphorylated by CK2 and ERK2, respectively.

Another advantage of the motif-centric approach is the specificity of the in vitro kinase reaction. Student's t-test (EGF/afatinib vs. motif-centric) revealed a low overlap of motifcentric peptides among the kinases (Figure 4d). Although ERK2, JNK1, p38a and CDK1 are all Pro-directed kinases, complementary profiles for the quantified motif-centric 
phosphorylation sites were observed (Figure 4d). The motif logos of these sites showed slight differences among the four Pro-directed kinases (Figure S7a). For example, the proportion of pT motifs was higher with $\mathrm{p} 38 \alpha$ kinase. In addition, more acidic amino acids were located at the C-terminal side of the phospho-acceptor site in the case of ERK2 kinase compared to CDK1 kinase. In addition to S/T sites, the pY motifs also differed between EGFR- and SRC-centric phosphopeptides (Figure S7b).

Further, from the two ratios (EGF vs. motif-centric and EGF/afatinib vs. motif-centric), we can estimate the stoichiometry of motif-centric phosphorylation sites (Figure 4e), assuming that the efficiency of in vitro kinase reaction is $100 \%$. The phosphorylation sites containing acidophilic kinase substrates targeted by CK2 generally exhibited higher phosphorylation stoichiometry than sites targeted by Pro-directed kinase and tyrosine kinases. The phosphorylation stoichiometry distribution that we observed here is consistent with our previous findings(Tsai et al., 2015). To further validate that the endogenous signals boosted by motif-centric peptides are lower than other peptide signals, the peak areas in the XICs of MS1 signals of quantified phosphopeptides were calculated based on the proportion of TMT intensity (Figure S8). The XICs of endogenous phosphopeptides boosted by the motif-centric peptides were lower than those of other phosphopeptides, which indicates the motif-centric approach is effective to identify these low-abundance kinase substrates.

In this motif-centered approach, a specific kinase can be chosen to target an endogenous peptide that is phosphorylated by that kinase. To confirm this, 8482 motif-centric phosphopeptides prepared with these seven kinases were subjected to KEGG pathway enrichment analysis using DAVID(Huang da et al., 2009) . The results showed that, as expected, known pathways involving the seven kinases, such as ErbB and insulin signaling, were enriched (Figure $5 \mathrm{a}$ and Table S2). We then performed STRING protein-protein interaction analysis of the 33 phosphoproteins comprising the ErbB pathway identified in this enrichment analysis (Figure 5b). Furthermore, we mapped the responsible kinases for the in vitro phosphorylation sites on these proteins (Figure $5 c$ ), showing how the kinases used for motifcentric peptides covered the targeted phosphosites within the targeted pathway. In other words, we can manipulate the targeted pathway by choosing the appropriate kinases, using our large-scale library of in vitro kinase-substrate relationships(Sugiyama et al., 2019). Overall, our findings indicate that the motif-centric approach can provide system-wide customizable maps consisting of targeted pathways under physiological or pathological regulation.

\section{Discussion}

The kinase assay-linked phosphoproteomics (KALIP) approach(Xue et al., 2013; Xue et al., 2012) has been developed to find potential kinase substrates. Bona fide direct substrates were found in the overlap of the in vitro phosphopeptides generated by kinase reaction with dephosphorylated peptides and in vivo kinase-dependent phosphorylation events in different LC-MS/MS runs. However, the dephosphorylation is not complete, and this may result in the false-positive identification of putative kinase substrates. Although stable-isotope KALIP(Xue et al., 2014) using ${ }^{18}$ O-ATP as a phosphate donor instead of ${ }^{16} \mathrm{O}$-ATP for in vitro kinase reaction 
improved the situation, the analytical throughput is still limited by the need for multiple LC/MS/MS analysis, and in addition the background effect due to incompletely dephosphorylated peptides reduces the detection sensitivity for putative kinase substrates. To tackle these challenges, we previously established a computational model (primary sequence preference, PSP score) to characterize the kinase sequence specificity toward substrate target sites based on known kinase-substrate relationships in order to exclude potential indirect targets of PKA (Imamura et al., 2017). Furthermore, the isobaric motifcentric approach developed in this study can link in vitro substrates and physiological phosphorylation events by monitoring both endogenous and back (specific kinase motiftargeting) phosphorylated signals in a single LC/MS/MS run without the need for a further dephosphorylation step, thereby enabling high-throughput analysis of putative kinase substrates. For example, the isobaric motif-centric approach was able to detect 266 CK2 motif-centric phosphorylation sites downregulated by CKi (Figure 2d). This method not only provides information about the fold-change between different samples, but also allows estimation of the stoichiometry of motif-centric phosphorylation sites (Figure 4e) based on the ratio between endogenous and motif-centric back phosphorylated signals, assuming that the efficiency of kinase reaction is $100 \%$.

Unlike metal affinity chromatography for enrichment of phosphopeptides, motif-specific immunoaffinity precipitation (IAP)-based LC/MS/MS makes it possible to recognize a characteristic sequence motif from a broad range of peptides by using different motif antibodies. Because each antibody binds phosphopeptides followed by a specific peptide motif, the overlap of identified phosphopeptides among different antibodies is low. The results of this approach are similar to those obtained with our motif targeting approach (Figure 4d), in which recognition between kinase and peptides is based on the specific sequence motif(Stokes et al., 2015; Sugiyama et al., 2019). The overlap between a given antibody and metal affinity chromatography ranged from roughly $16 \%$ with the all Ser/Thr antibody mix, to a low of only 3.6\% with phosphotyrosine pY-1000 (Stokes et al., 2015). Anthony et al. aimed to enlarge the phosphoproteome coverage by using both $\mathrm{TiO}_{2}$ followed by basic $\mathrm{pH}$ reversed-phase fractionation and motif-specific IAP with four different phosphorylation motif-specific antibodies(Possemato et al., 2017). In total, 8947 nonredundant peptides were identified in the $\mathrm{TiO}_{2}$ data set, of which only 852 (9.5\%) were in common with the peptides identified in the IAPs. These results suggest that the range of phosphorylation within a given system is so broad that no single approach is likely to provide comprehensive coverage.

In the case of IAPs, the specificity of the antibodies is not high enough to distinguish phosphopeptides with similar motifs; for example, MAPK phosphorylates substrates with the consensus sequence PX(S/T)P and CDKs phosphorylate substrates containing the consensus sequence (S/T)PXR/K(Songyang et al., 1996). However, the complementary regulated motifcentric phosphorylation sites (Figure $4 \mathrm{~d}$ and Figure S7) were differentiated in this study, even though the kinases all belong to the same Pro-directed kinase group. The motif-centric approach was also able to distinguish the motif difference (PX(S/T)P and (S/T)PXR/K) for ERK2 
and CDK1 (Figure 4d). In addition, the purification specificity in IAP (less than 50\%) is much lower than that in the metal affinity-based approach (Possemato et al., 2017), which means that mg amounts of starting materials are necessary for the IAPs method. For our isobaric motif-centric approach, we performed metal affinity chromatography (IMAC in this study) after motif-centric enrichment, and obtained a phosphopeptides purification specificity of over 90\% (Figure S9). In addition, the IAPs approach uses selected antibodies to isolate targeting peptides, and this may cause unnecessary sample loss during the purification step. In contrast, the motif-centric approach uses kinase to recognize specific substrates and transfer the phosphate group to back-phosphorylated peptides. Endogenous phosphopeptides, especially low-abundance tyrosine phosphopeptides, are not removed before IMAC enrichment. Therefore, only a few tens of $\mu \mathrm{g}$ of material was required for our isobaric motif-centric approach. Recently, a novel antibody-based method, called PTMScan Direct, was developed for the identification and quantitation of peptides derived from proteins that are critical signaling nodes of various pathways (Stokes et al., 2012). However, the coverage of the IAPs approach is still limited by the availability and quality of antibodies. In contrast, recombinant active kinases are much more readily available than antibodies. In our previous study (Sugiyama et al., 2019), we were able to identify a total of 175,574 potential direct kinase substrates by using 385 active kinases ( 354 wild-type protein kinases, 21 mutants and 10 lipid kinases). Based on this kinase substrate library, it is easily possible to select multiple kinases for targeting pathway analysis.

The multiplexing nature of isobaric labeling is particularly useful to achieve higher sensitivity with limited individual sample amounts, as in the case of tyrosine phosphopeptides. Chua et al. (Chua et al., 2020) and Fang et al. (Fang et al., 2020) used samples with and without pervanadate (tyrosine phosphatase inhibitor)-treated cells as a boosting channel to increase the relative abundance of tyrosine phosphorylation sites. Although around 2300 (Chua et al., 2020) and $835 \mathrm{pY}$ phosphopeptides (Fang et al., 2020) were detected, the required amount of starting material is at the mg level. In addition, the usage of antibodies for $\mathrm{pY}$ phosphopeptides enrichment is also necessary for their approach. Here, in contrast, we generate higher-purity tyrosine phosphopeptides via in vitro kinase reaction (motif-centric). By using motif-centric peptides, we could detect up to 7129 (SRC targeting) and 7280 (EGFR targeting) tyrosine phosphopeptides without the need for immunoprecipitation, using only a few tens of $\mu \mathrm{g}$ of starting materials.

\section{Limitations}

Although the performance of isobaric labeling-based quantitative approaches is affected by co-selected precursor ions, the usage of SPS-MS ${ }^{3}$ can minimize this effect and improve the detection sensitivity by utilizing co-fragmented multiple (up to 10) $\mathrm{MS}^{2}$ fragment ions with higher intensity (McAlister et al., 2014). Moreover, the newly available Real Time Search-MS ${ }^{3}$ method (RTS-MS ${ }^{3}$ ) (Erickson et al., 2019) or ion mobility technique (Bekker-Jensen et al., 2020; Hebert et al., 2018; Ogata and Ishihama, 2020) provides a solution for precise and accurate quantitation without sacrificing proteome coverage. Recently, the use of spiked TMT labeling 
peptides with boosting or carrier samples has reduced the TMT reporter ions dynamic range and decreased the quantitation accuracy (Tsai et al., 2020). Cheung et al. (Cheung et al., 2021) and Stopfer et al. (Stopfer et al., 2021a) also demonstrated that an increase in carrier proteome level requires a concomitant increase in the number of ions sampled to maintain quantitative accuracy. Therefore, it will be important to optimize the spiking amount of carrier/boosting and the TMT channel design for the isobaric motif-centric strategy. Optimization of the MS parameters to improve the ion sampling by adjusting the ion injection time and AGC will also be beneficial to improve the quantitation performance(Cheung et al., 2021; Tsai et al., 2020). To overcome the challenge presented by the large quantitation dynamic range, it may be useful to integrate the motif-centric approach with isotope-based SureQuant (Stopfer et al., 2021b) or Internal Standard Triggered-Parallel Reaction Monitoring (IS-PRM) (Gallien et al., 2015) quantitation.

\section{Conclusions}

The isobaric motif-centric strategy presented here can be used to enhance the sensitivity of specific kinase downstream signaling analysis, especially for tyrosine phosphopeptides. It provides a simple yet highly effective quantitative phosphoproteomic workflow suitable for multiplexed analysis of relatively small biological or clinical samples (sub $\mathrm{mg}$ ), including cells or tissues. This approach enables the quantitation of both fold-change and stoichiometry among thousands of phosphopeptides generated by specific kinases in signaling pathways. The usage of multiple kinases for motif targeting analysis effectively increases the phosphoproteome coverage. Overall, we anticipate this strategy should find broad biomedical applications for targeting kinase/pathway analysis where limited amounts of starting cells or tissues are available.

\section{Acknowledgement}

We would like to thank all lab members for fruitful discussions. C.-F.T. was supported by a JSPS Grant-in-Aid for a postdoctoral fellowship for overseas researchers (15F15343). This work was supported by the JST Strategic Basic Research Program, CREST (grant No. 18070870) to Y. I., and by a JSPS Grants-in-Aid for Scientific Research (No. 17H03605, No. $21 \mathrm{H02459}$ to Y. I., No. $20 \mathrm{~K} 21478$ to Y. I. and K. O., and No. $20 \mathrm{HO} 4845$, No. $21 \mathrm{HO2466}$ to N. S.)

\section{Author Contributions}

Conceptualization, C.F.T. and Y.I.; Methodology, C.F.T. and Y.I.; Investigation, C.F.T., K.O. and N. S.; Writing - Original Draft, C.F.T.; Writing -Review \& Editing, C.F.T. and Y.I; Funding Acquisition, Y.I.; Supervision, Y.I. 


\section{Declaration of Interests}

The authors declare no competing interests.

Figure

(a) Samples
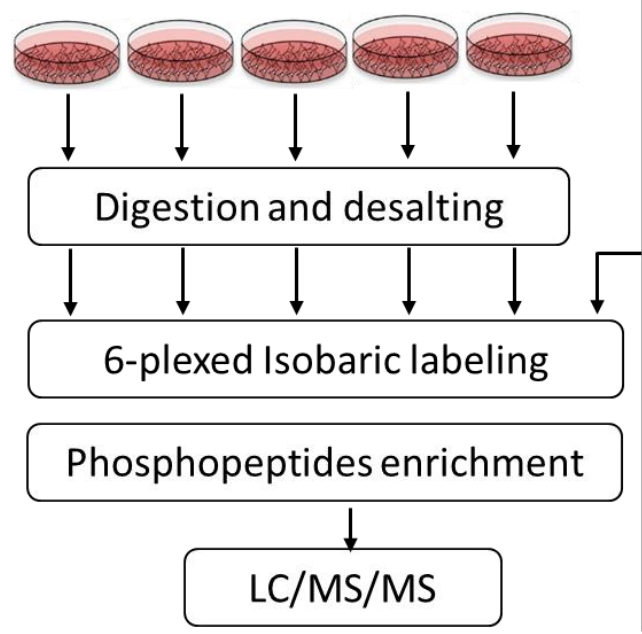

\section{Preparation of motif-centric peptides}

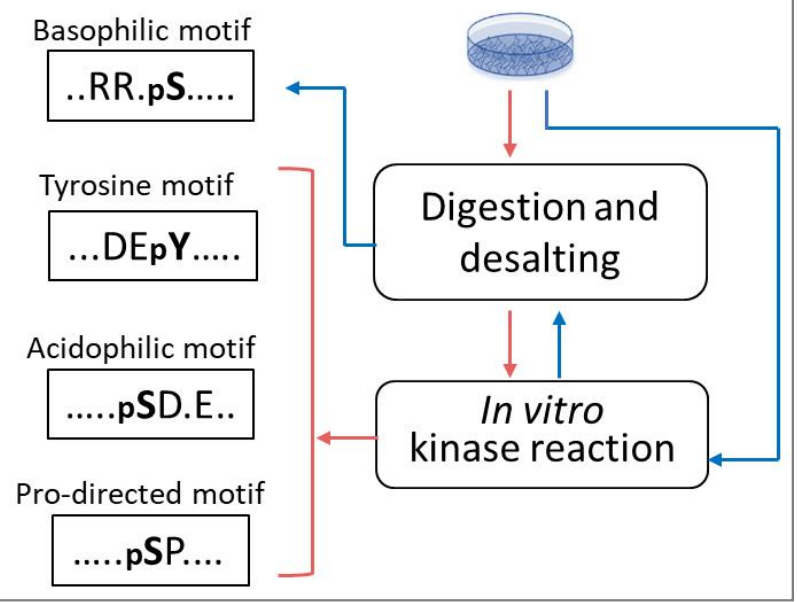

(b)

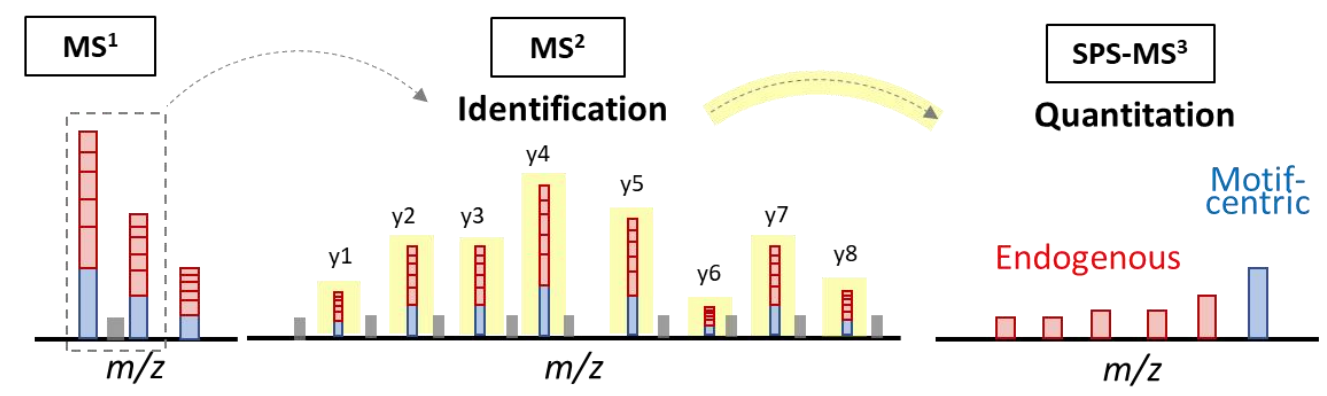

Figure 1. Workflow of isobaric motif-centric phosphoproteomics with in vitro kinase reaction. (a) The motif-centric peptides were generated by in vitro kinase reaction. Tryptic peptides from study samples and motif-centric peptides were labeled with different isobaric tags (TMT in this study). After mixing, the TMT-labeled phosphopeptides were enriched and analyzed by LC/MS/MS. (b) TMT-labeled ions from endogenous and motif-centric phosphopeptides are assembled as a single peak at the $\mathrm{MS}^{1}$ level and then fragmented at the $\mathrm{MS}^{2}$ level to identify the peptide sequence. Relative quantification of endogenous phosphopeptides between different samples is achieved by reporter ions at the $\mathrm{MS}^{3}$ level. 
(a)

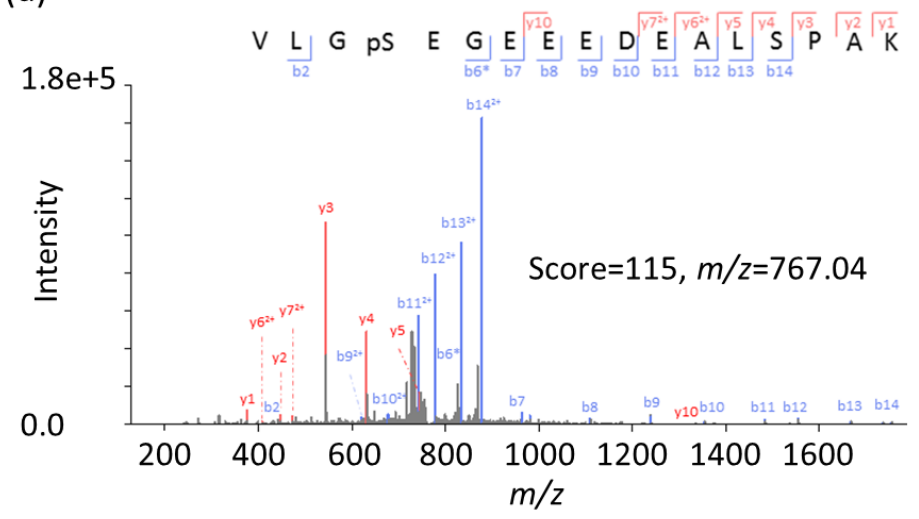

(c)

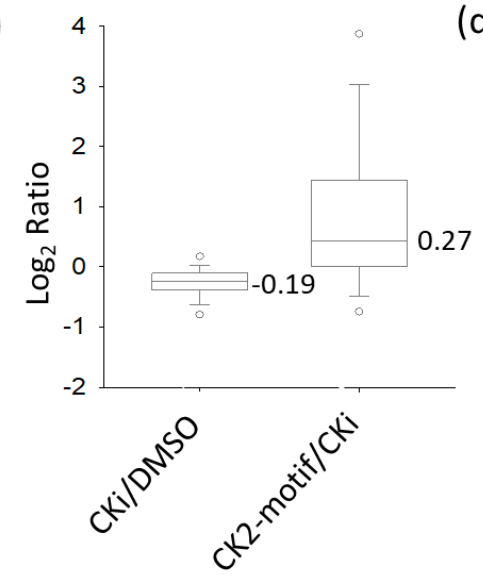

(b)

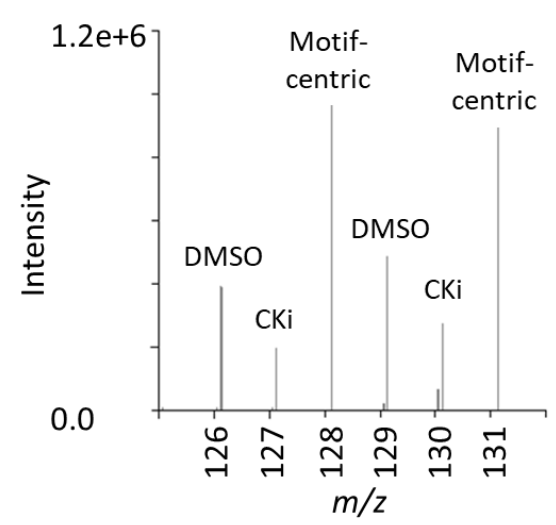

Figure 2. Typical example of the isobaric CK2 motif-centric phosphoproteomic approach.

(a) An MS/MS spectrum of TMT-labeled phosphopeptide (VLGpSEGEEEDEALSPAK, triply charged). Product ions at the $\mathrm{MS}^{2}$ level identified the peptide sequence and phosphorylation site localization. (b) An MS/MS/MS spectrum at the 6-plexed TMT reporter ion region. Three samples (DMSO, CKi-treated and CK2 motif-centric phosphopeptides) in duplicate preparations, labeled with 6-plexed TMT reagents, were quantified at the $\mathrm{MS}^{3}$ level. (c) The ratio distribution on a $\log _{2}$ scale of identified phosphopeptides. Left bar: CKi/DMSO, right bar: motif-centric/CKi. (d) Quantitation result for CKi treatment. Sequence motif analysis for the down-regulated phosphorylation sites after CKi treatment was performed. The blue bar indicates the amount of phosphorylation sites significantly increased after in vitro kinase reaction (CK2-motif centric peptides). (e) The $\log _{2}$ ratio correlation between CKi/DMSO and motif-centric/CKi. The red and green circles mean up-regulated and down-regulated phosphorylation sites after CKi treatment, respectively, in panel (d). The blue fill in the green circle indicates downregulated CK2-motif centric peptides in panel (d). 

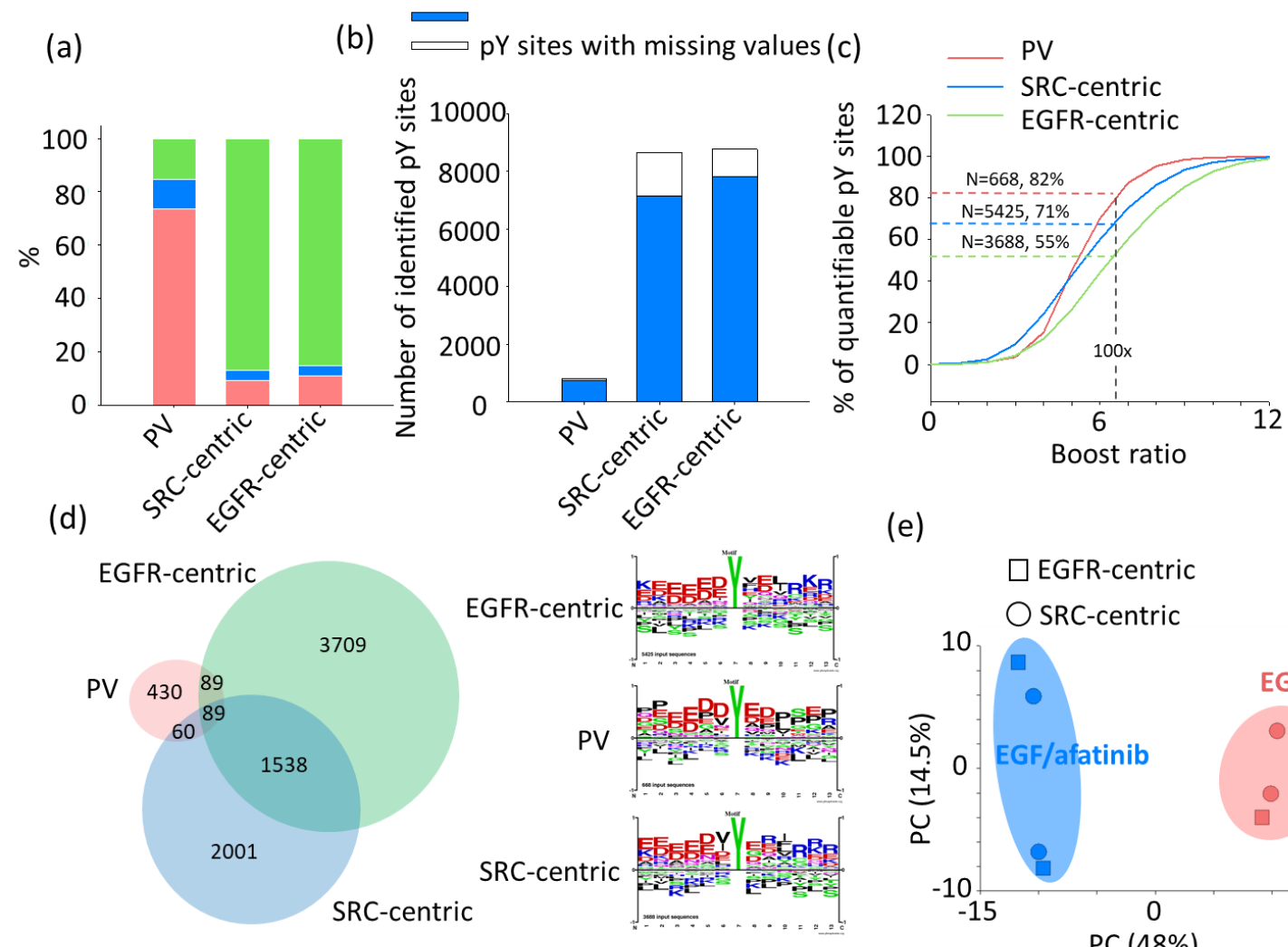

(e)

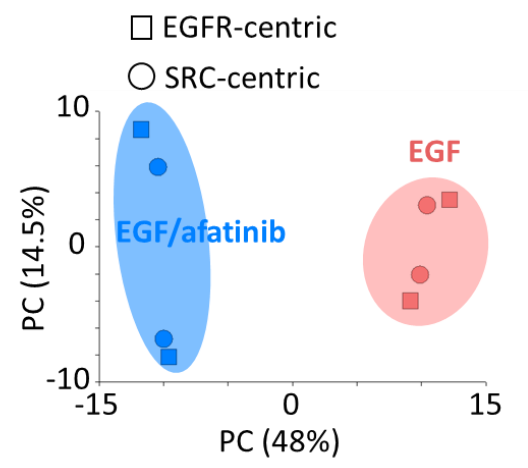

Figure 3. Summary of pY motif-centric phosphoproteomic approach. (a) The content (\%) of identified $\mathrm{pY}$ sites using PV-treated, SRC and EGFR phosphorylated peptides as the $\mathrm{pY}$-centric samples. (b) The number of $\mathrm{pY}$ sites identified in each experiment. A quantifiable site is defined as a pY site having signal intensities in at least 2 TMT reporter channels. Otherwise, pY sites were considered as missing values. (c) The content of quantifiable $p Y$ sites filtered by boosting ratio, defined as the TMT signal at $m / z 131$ divided by the averaged signal at $\mathrm{m} / \mathrm{z}$ 127 and 129. (d) The overlap and the sequence logos of boosting ratio-filtered quantified $p Y$ sites between different motif-centric approaches (e) The PCA analysis of commonly quantified pY sites (filtered). 


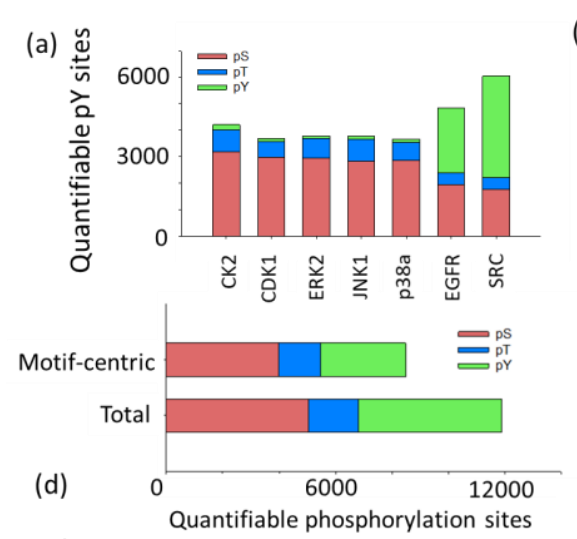

$\mathrm{pS} / \mathrm{pT}$

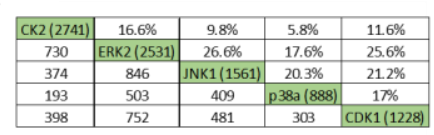

pY

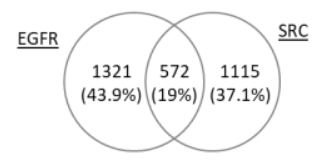

(b)

$\log _{2}$ ratio (EGF vs EGF/afatinib)

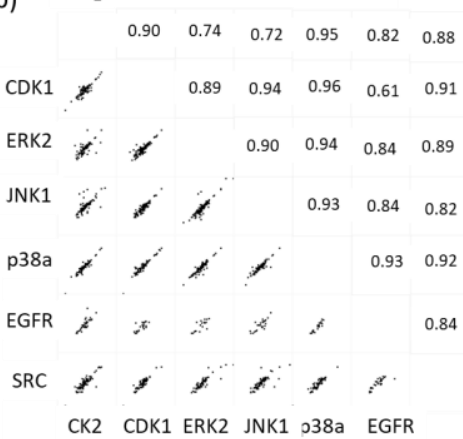

(e)

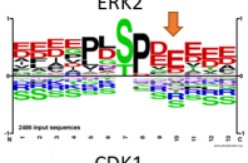

CDK1

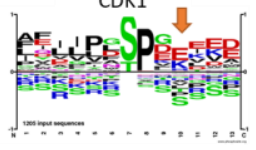

$\square$ Quantifiable

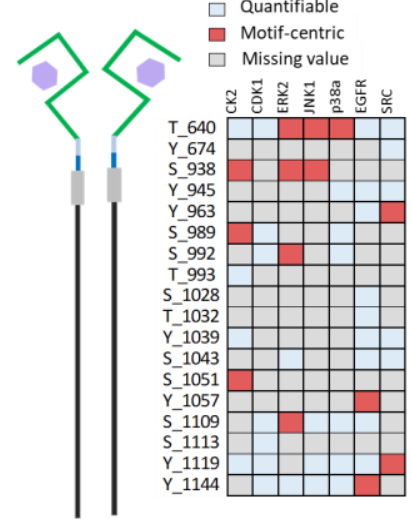

CDK1

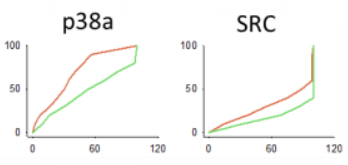

EGFR

- EGF

JNK1

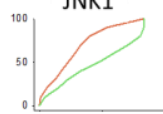

Cumulative fraction $(\%)$

Figure 4. Summary of motif-centric phosphoproteomic approach with multiple kinases. (a) The number of quantified phosphorylation sites (class 1) obtained using different kinases. (b) The ratio correlation of commonly regulated phosphorylation sites (EGF vs. EGF/afatinib, $p<0.05$ ) in experiments with different kinases. (c) The motif-centric sites (red color) among quantified phosphorylation sites in EGFR. (d) The overlap of motif-centric phosphorylation sites between the samples with different kinases as motif-centric samples. The sequence motif analysis for the ERK2 and CDK1 centric phosphorylation sites. (e) The phosphorylation stoichiometry distribution of motif-centric phosphorylation sites after afatinib treatment. 
(a)

(c)

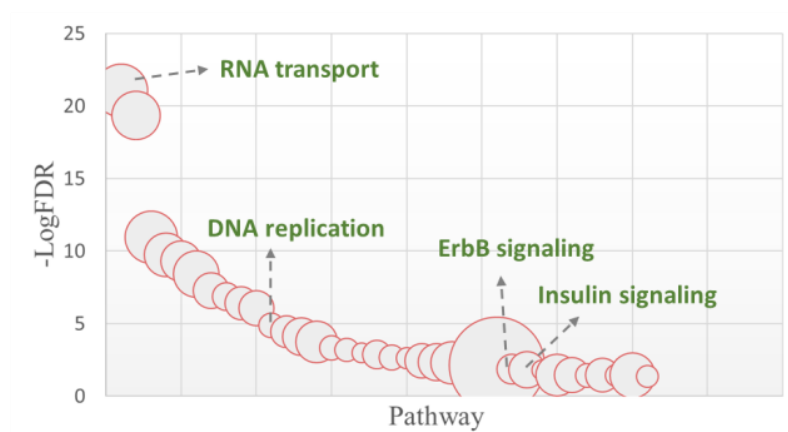

(b)

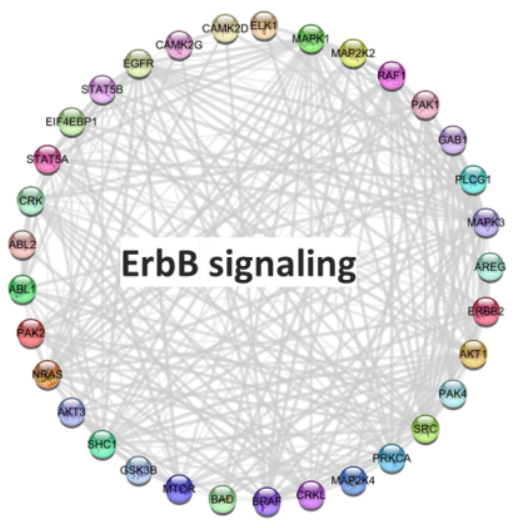

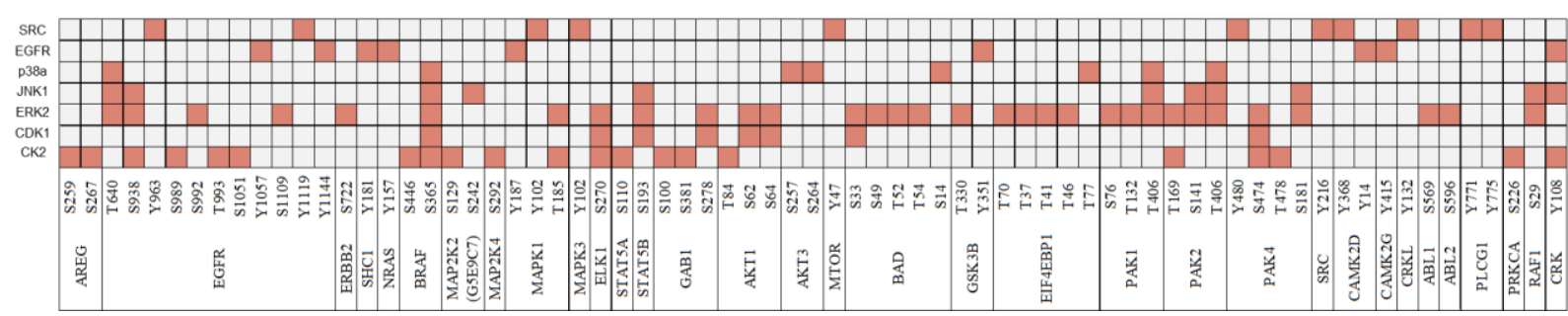

Figure 5. Pathway enrichment analysis of motif-centric phosphorylated sites with multiple kinases. (a) KEGG pathway enrichment analysis by DAVID. (b) STRING protein-protein interaction analysis of the phosphoproteins enriched in the ErbB signaling pathway. (c) Phosphorylation sites on proteins in the ErbB signaling pathway and the corresponding kinases. 


\section{Reference}

Abe, Y., Nagano, M., Tada, A., Adachi, J., and Tomonaga, T. (2017). Deep Phosphotyrosine Proteomics by Optimization of Phosphotyrosine Enrichment and MS/MS Parameters. J Proteome Res 16, 1077-1086.

Bekker-Jensen, D.B., Kelstrup, C.D., Batth, T.S., Larsen, S.C., Haldrup, C., Bramsen, J.B., Sorensen, K.D., Hoyer, S., Orntoft, T.F., Andersen, C.L., et al. (2017). An Optimized Shotgun Strategy for the Rapid Generation of Comprehensive Human Proteomes. Cell Syst 4, 587-599 e584.

Bekker-Jensen, D.B., Martinez-Val, A., Steigerwald, S., Ruther, P., Fort, K.L., Arrey, T.N., Harder, A., Makarov, A., and Olsen, J.V. (2020). A Compact Quadrupole-Orbitrap Mass Spectrometer with FAIMS Interface Improves Proteome Coverage in Short LC Gradients. Mol Cell Proteomics 19, 716-729.

Bian, Y., Li, L., Dong, M., Liu, X., Kaneko, T., Cheng, K., Liu, H., Voss, C., Cao, X., Wang, Y., et al. (2016). Ultra-deep tyrosine phosphoproteomics enabled by a phosphotyrosine superbinder. Nat Chem Biol 12, 959-966.

Bloom, J., and Cross, F.R. (2007). Multiple levels of cyclin specificity in cell-cycle control. Nat Rev Mol Cell Biol 8, 149-160.

Budnik, B., Levy, E., Harmange, G., and Slavov, N. (2018). SCoPE-MS: mass spectrometry of single mammalian cells quantifies proteome heterogeneity during cell differentiation.

Genome Biol 19, 161.

Cheung, T.K., Lee, C.Y., Bayer, F.P., McCoy, A., Kuster, B., and Rose, C.M. (2021). Defining the carrier proteome limit for single-cell proteomics. Nat Methods 18, 76-83. Chon, H.J., Bae, K.J., Lee, Y., and Kim, J. (2015). The casein kinase 2 inhibitor, CX-4945, as an anti-cancer drug in treatment of human hematological malignancies. Front Pharmacol 6,70 .

Chua, X.Y., Mensah, T., Aballo, T., Mackintosh, S.G., Edmondson, R.D., and Salomon, A.R. (2020). Tandem Mass Tag Approach Utilizing Pervanadate BOOST Channels Delivers Deeper Quantitative Characterization of the Tyrosine Phosphoproteome. Mol Cell Proteomics 19, 730-743.

Cohen, P. (2002). The origins of protein phosphorylation. Nat Cell Biol 4, E127-130.

Cox, J., and Mann, M. (2008). MaxQuant enables high peptide identification rates, individualized p.p.b.-range mass accuracies and proteome-wide protein quantification. Nat Biotechnol 26, 1367-1372.

Dong, M., Bian, Y., Wang, Y., Dong, J., Yao, Y., Deng, Z., Qin, H., Zou, H., and Ye, M. (2017). Sensitive, Robust, and Cost-Effective Approach for Tyrosine Phosphoproteome Analysis. Anal Chem 89, 9307-9314.

Dou, M., Clair, G., Tsai, C.F., Xu, K., Chrisler, W.B., Sontag, R.L., Zhao, R., Moore, R.J., Liu, T., Pasa-Tolic, L., et al. (2019). High-Throughput Single Cell Proteomics Enabled by Multiplex Isobaric Labeling in a Nanodroplet Sample Preparation Platform. Anal Chem 91, 13119-13127.

Erickson, B.K., Mintseris, J., Schweppe, D.K., Navarrete-Perea, J., Erickson, A.R., Nusinow, D.P., Paulo, J.A., and Gygi, S.P. (2019). Active Instrument Engagement Combined with a Real-Time Database Search for Improved Performance of Sample Multiplexing Workflows. J Proteome Res 18, 1299-1306.

Fang, B., Izumi, V., Rix, L.L.R., Welsh, E., Pike, I., Reuther, G.W., Haura, E.B., Rix, U., and Koomen, J.M. (2020). Lowering Sample Requirements to Study Tyrosine Kinase Signaling Using Phosphoproteomics with the TMT Calibrator Approach. Proteomics 20, e2000116. Gallien, S., Kim, S.Y., and Domon, B. (2015). Large-Scale Targeted Proteomics Using Internal Standard Triggered-Parallel Reaction Monitoring (IS-PRM). Mol Cell Proteomics 14, 1630-1644. 
Hebert, A.S., Prasad, S., Belford, M.W., Bailey, D.J., McAlister, G.C., Abbatiello, S.E., Huguet, R., Wouters, E.R., Dunyach, J.J., Brademan, D.R., et al. (2018). Comprehensive Single-Shot Proteomics with FAIMS on a Hybrid Orbitrap Mass Spectrometer. Anal Chem 90, 9529-9537.

Hogrebe, A., von Stechow, L., Bekker-Jensen, D.B., Weinert, B.T., Kelstrup, C.D., and Olsen, J.V. (2018). Benchmarking common quantification strategies for large-scale phosphoproteomics. Nat Commun 9, 1045.

Hornbeck, P.V., Zhang, B., Murray, B., Kornhauser, J.M., Latham, V., and Skrzypek, E. (2015). PhosphoSitePlus, 2014: mutations, PTMs and recalibrations. Nucleic Acids Res 43, D512-520.

Huang da, W., Sherman, B.T., and Lempicki, R.A. (2009). Systematic and integrative analysis of large gene lists using DAVID bioinformatics resources. Nat Protoc 4, 44-57. Hughes, C.S., Zhu, C., Spicer, V., Krokhin, O.V., and Morin, G.B. (2017). Evaluating the Characteristics of Reporter Ion Signal Acquired in the Orbitrap Analyzer for Isobaric Mass Tag Proteome Quantification Experiments. J Proteome Res 16, 1831-1838.

Hunter, T. (2000). Signaling--2000 and beyond. Cell 100, 113-127.

Hunter, T. (2009). Tyrosine phosphorylation: thirty years and counting. Curr Opin Cell Biol 21, 140-146.

Hunter, T., and Sefton, B.M. (1980). Transforming gene product of Rous sarcoma virus phosphorylates tyrosine. Proc Natl Acad Sci U S A 77, 1311-1315.

Imamura, H., Sugiyama, N., Wakabayashi, M., and Ishihama, Y. (2014). Large-scale identification of phosphorylation sites for profiling protein kinase selectivity. J Proteome Res 13, 3410-3419.

Imamura, H., Wagih, O., Niinae, T., Sugiyama, N., Beltrao, P., and Ishihama, Y. (2017). Identifications of Putative PKA Substrates with Quantitative Phosphoproteomics and Primary-Sequence-Based Scoring. J Proteome Res 16, 1825-1830.

Iwasaki, M., Miwa, S., Ikegami, T., Tomita, M., Tanaka, N., and Ishihama, Y. (2010). Onedimensional capillary liquid chromatographic separation coupled with tandem mass spectrometry unveils the Escherichia coli proteome on a microarray scale. Anal Chem 82 , 2616-2620.

Li, X., Cox, J.T., Huang, W., Kane, M., Tang, K., and Bieberich, C.J. (2016). Quantifying Kinase-Specific Phosphorylation Stoichiometry Using Stable Isotope Labeling In a Reverse In-Gel Kinase Assay. Anal Chem 88, 11468-11475.

Masuda, T., Tomita, M., and Ishihama, Y. (2008). Phase transfer surfactant-aided trypsin digestion for membrane proteome analysis. J Proteome Res 7, 731-740.

McAlister, G.C., Nusinow, D.P., Jedrychowski, M.P., Wuhr, M., Huttlin, E.L., Erickson, B.K., Rad, R., Haas, W., and Gygi, S.P. (2014). MultiNotch MS3 enables accurate, sensitive, and multiplexed detection of differential expression across cancer cell line proteomes. Anal Chem 86, 7150-7158.

Mertins, P., Tang, L.C., Krug, K., Clark, D.J., Gritsenko, M.A., Chen, L., Clauser, K.R., Clauss, T.R., Shah, P., Gillette, M.A., et al. (2018). Reproducible workflow for multiplexed deep-scale proteome and phosphoproteome analysis of tumor tissues by liquid chromatography-mass spectrometry. Nat Protoc 13, 1632-1661.

Moriya, Y., Kawano, S., Okuda, S., Watanabe, Y., Matsumoto, M., Takami, T., Kobayashi, D., Yamanouchi, Y., Araki, N., Yoshizawa, A.C., et al. (2019). The jPOST environment: an integrated proteomics data repository and database. Nucleic Acids Res 47, D1218-D1224. Mundina-Weilenmann, C., Chang, C.F., Gutierrez, L.M., and Hosey, M.M. (1991).

Demonstration of the phosphorylation of dihydropyridine-sensitive calcium channels in chick skeletal muscle and the resultant activation of the channels after reconstitution. J Biol Chem 266, 4067-4073.

O'Shea, J.P., Chou, M.F., Quader, S.A., Ryan, J.K., Church, G.M., and Schwartz, D. (2013). pLogo: a probabilistic approach to visualizing sequence motifs. Nat Methods 10, 1211-1212. Ogata, K., and Ishihama, Y. (2020). Extending the Separation Space with Trapped Ion Mobility Spectrometry Improves the Accuracy of Isobaric Tag-Based Quantitation in Proteomic LC/MS/MS. Anal Chem 92, 8037-8040. 
Possemato, A.P., Paulo, J.A., Mulhern, D., Guo, A., Gygi, S.P., and Beausoleil, S.A. (2017). Multiplexed Phosphoproteomic Profiling Using Titanium Dioxide and Immunoaffinity Enrichments Reveals Complementary Phosphorylation Events. J Proteome Res 16, 15061514.

Rappsilber, J., Mann, M., and Ishihama, Y. (2007). Protocol for micro-purification, enrichment, pre-fractionation and storage of peptides for proteomics using StageTips. Nat Protoc 2, 1896-1906.

Russo, G.L., Vandenberg, M.T., Yu, I.J., Bae, Y.S., Franza, B.R., Jr., and Marshak, D.R. (1992). Casein kinase II phosphorylates p34cdc2 kinase in G1 phase of the HeLa cell division cycle. J Biol Chem 267, 20317-20325.

Sharma, K., D'Souza, R.C., Tyanova, S., Schaab, C., Wisniewski, J.R., Cox, J., and Mann, M. (2014). Ultradeep human phosphoproteome reveals a distinct regulatory nature of Tyr and Ser/Thr-based signaling. Cell Rep 8, 1583-1594.

Songyang, Z., Lu, K.P., Kwon, Y.T., Tsai, L.H., Filhol, O., Cochet, C., Brickey, D.A., Soderling, T.R., Bartleson, C., Graves, D.J., et al. (1996). A structural basis for substrate specificities of protein Ser/Thr kinases: primary sequence preference of casein kinases I and II, NIMA, phosphorylase kinase, calmodulin-dependent kinase II, CDK5, and Erk1. Mol Cell Biol 16, 6486-6493.

Stokes, M.P., Farnsworth, C.L., Gu, H., Jia, X., Worsfold, C.R., Yang, V., Ren, J.M., Lee, K.A., and Silva, J.C. (2015). Complementary PTM Profiling of Drug Response in Human Gastric Carcinoma by Immunoaffinity and IMAC Methods with Total Proteome Analysis. Proteomes 3, 160-183.

Stokes, M.P., Farnsworth, C.L., Moritz, A., Silva, J.C., Jia, X., Lee, K.A., Guo, A., Polakiewicz, R.D., and Comb, M.J. (2012). PTMScan direct: identification and quantification of peptides from critical signaling proteins by immunoaffinity enrichment coupled with LCMS/MS. Mol Cell Proteomics 11, 187-201.

Stopfer, L.E., Conage-Pough, J.E., and White, F.M. (2021a). Quantitative Consequences of Protein Carriers in Immunopeptidomics and Tyrosine Phosphorylation MS(2) Analyses. Mol Cell Proteomics 20, 100104.

Stopfer, L.E., Flower, C.T., Gajadhar, A.S., Patel, B., Gallien, S., Lopez-Ferrer, D., and White, F.M. (2021b). High-Density, Targeted Monitoring of Tyrosine Phosphorylation Reveals Activated Signaling Networks in Human Tumors. Cancer Res 81, 2495-2509. Sugiyama, N., Imamura, H., and Ishihama, Y. (2019). Large-scale Discovery of Substrates of the Human Kinome. Sci Rep 9, 10503.

Szklarczyk, D., Gable, A.L., Lyon, D., Junge, A., Wyder, S., Huerta-Cepas, J., Simonovic, M., Doncheva, N.T., Morris, J.H., Bork, P., et al. (2019). STRING v11: protein-protein association networks with increased coverage, supporting functional discovery in genomewide experimental datasets. Nucleic Acids Res 47, D607-D613.

Thompson, A., Schafer, J., Kuhn, K., Kienle, S., Schwarz, J., Schmidt, G., Neumann, T., Johnstone, R., Mohammed, A.K., and Hamon, C. (2003). Tandem mass tags: a novel quantification strategy for comparative analysis of complex protein mixtures by MS/MS. Anal Chem 75, 1895-1904.

Tsai, C.F., Hsu, C.C., Hung, J.N., Wang, Y.T., Choong, W.K., Zeng, M.Y., Lin, P.Y., Hong, R.W., Sung, T.Y., and Chen, Y.J. (2014). Sequential phosphoproteomic enrichment through complementary metal-directed immobilized metal ion affinity chromatography. Anal Chem 86, 685-693.

Tsai, C.F., Wang, Y.T., Yen, H.Y., Tsou, C.C., Ku, W.C., Lin, P.Y., Chen, H.Y., Nesvizhskii, A.I., Ishihama, Y., and Chen, Y.J. (2015). Large-scale determination of absolute phosphorylation stoichiometries in human cells by motif-targeting quantitative proteomics. Nat Commun 6, 6622.

Tsai, C.F., Zhao, R., Williams, S.M., Moore, R.J., Schultz, K., Chrisler, W.B., Pasa-Tolic, L., Rodland, K.D., Smith, R.D., Shi, T., et al. (2020). An Improved Boosting to Amplify Signal with Isobaric Labeling (iBASIL) Strategy for Precise Quantitative Single-cell Proteomics. Mol Cell Proteomics 19, 828-838. 
Tyanova, S., Temu, T., and Cox, J. (2016a). The MaxQuant computational platform for mass spectrometry-based shotgun proteomics. Nat Protoc 11, 2301-2319.

Tyanova, S., Temu, T., Sinitcyn, P., Carlson, A., Hein, M.Y., Geiger, T., Mann, M., and Cox, J. (2016b). The Perseus computational platform for comprehensive analysis of (prote)omics data. Nat Methods 13, 731-740.

Villen, J., Beausoleil, S.A., Gerber, S.A., and Gygi, S.P. (2007). Large-scale phosphorylation analysis of mouse liver. Proc Natl Acad Sci U S A 104, 1488-1493.

Wang, Y.T., Pan, S.H., Tsai, C.F., Kuo, T.C., Hsu, Y.L., Yen, H.Y., Choong, W.K., Wu, H.Y., Liao, Y.C., Hong, T.M., et al. (2017). Phosphoproteomics Reveals HMGA1, a CK2

Substrate, as a Drug-Resistant Target in Non-Small Cell Lung Cancer. Sci Rep 7, 44021.

Xue, L., Geahlen, R.L., and Tao, W.A. (2013). Identification of direct tyrosine kinase substrates based on protein kinase assay-linked phosphoproteomics. Mol Cell Proteomics 12, 2969-2980.

Xue, L., Wang, P., Cao, P., Zhu, J.K., and Tao, W.A. (2014). Identification of extracellular signal-regulated kinase 1 (ERK1) direct substrates using stable isotope labeled kinase assay-linked phosphoproteomics. Mol Cell Proteomics 13, 3199-3210.

Xue, L., Wang, W.H., Iliuk, A., Hu, L., Galan, J.A., Yu, S., Hans, M., Geahlen, R.L., and Tao, W.A. (2012). Sensitive kinase assay linked with phosphoproteomics for identifying direct kinase substrates. Proc Natl Acad Sci U S A 109, 5615-5620. 